\title{
Fabrication of Pt- and Au-coated W Nano Tip with Electroplated Films as a Noble-metal Source toward Viable Application for Long-life Electron Source
}

\author{
Hirotaka Asai, ${ }^{\dagger}$ Takayuki Tanaka, Hidekazu Murata, Eiji Rokuta \\ Faculty of Science and Technology, Meijo University, Shiogamaguchi, Tempaku, Nagoya 468-8502, Japan \\ $\dagger$ Corresponding author: 163441501@ccalumni.meijo-u.ac.jp
}

Received: 26 May, 2021; Accepted: 20 December, 2021; Published: 28 December, 2021

The surface diffusion method was used to produce tungsten (W) nanopyramids coated with monolayer films of platinum (Pt) and gold $(\mathrm{Au})$. Although the general structures of the nanopyramids were the same, the top of the nanopyramids was different from the $\mathrm{Pt}$ and $\mathrm{Au}$ cases, i.e., a monomer was observed for Pt, whereas a trimer observed for Au. The effects of simultaneous heating and the electric field on the structural evolution of nanopyramids were also studied. We observed that the broken nanopyramids were rapidly regenerated by heating in an electric field. The opening angles of field-emitted electrons were found to be approximately $\pm 2^{\circ}$ and $\pm 2.5^{\circ}$ for Pt and $\mathrm{Au}$, respectively. From the obtained results, it was observed that the field-emission properties of all specimens were almost the same.

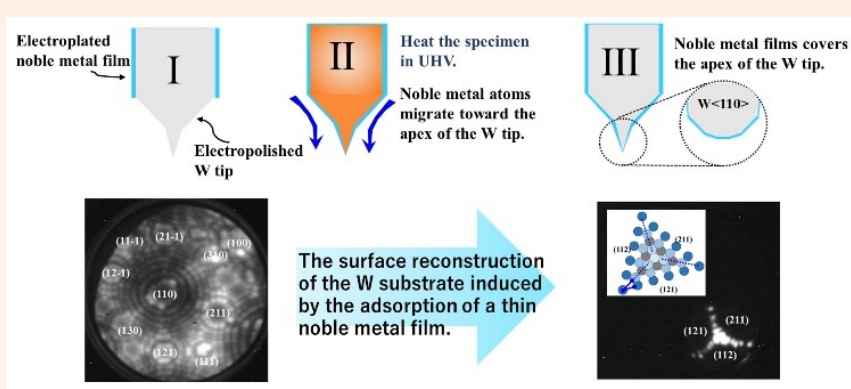

Keywords Nano tip; Electron source; Gas field ion source, field ion microscopy; Faceting; Tungsten

\section{INTRODUCTION}

In scanning electron microscopes and transmission electron microscopes, electron sources are crucial. The development of next-generation electron sources is crucial for improving the efficiency of electron microscopes. Materials such as strained superlattice GaAs/GaAsP semiconductor photocathodes [1], graphene/h-BN/n-Si heterostructure planar electron emission devices [2], $\mathrm{Co}_{2} \mathrm{MnGa}\langle 100\rangle$ tip [3], $\mathrm{LaB}_{6}\langle 100\rangle$ nanowire [4], $\mathrm{CeB}_{6}\langle 310\rangle$ tip [5], and amorphous carbon-coated metal tip $[6,7]$ have been investigated in this direction. One of the important factors that determines the efficiency (probe diameter or coherence) of an electron optical device is the brightness of the electron source. Shimoyama estimated the theoretically feasible maximum brightness $\beta_{0}\left(\mathrm{~A} \mathrm{~cm}^{-2} \mathrm{eV}^{-1} \mathrm{sr}^{-1}\right)$ of the electron optics system by using the formula of $\beta_{0}=J_{0} / \pi d$, where $J_{0}$ is the current density at the electron source's surface and $d$ is the mean tangential energy spread of field-emitted electrons [8,9]. Therefore, one of the basic strategies for making more progress in electron sources is to shrink the apex regions (note that the term $\pi d \sim 1$ because the value of $d$ is $0.2 \mathrm{eV}$ to 0.4 $\mathrm{eV})$.

The single-atom tip, which was first obtained by Fink in 1986 [10], is the sharpest of all metal tips reported so far. In the late 1980s and early 2000s, many researchers employed various techniques that produced atomically sharp tips [11-17]. However, these tips are not commercially acceptable, essentially because of their poor thermodynamical stability. A tungsten (W) pyramid (nanopyramid) coated with a monolayer film of noble metals (NMs) is a compatible atomically sharp tip feasible for application. A schematic of the NM-coated nanopyramid formed on the $\mathrm{W}(110)$ tip is shown in Figure 1(a). The basic properties of nanopyramid were investigated experimentally and theoretically in the 1990s by Madey et al. [18-22]. The NM adsorbates largely reduce the surface free energy of $\{211\}$ faces and enhance the surface energy anisotropy [23]. Consequently, the faceting phenomenon of $\mathrm{W}$ surface adsorbed onto the monolayer films of NM atoms yielded the three large sides of equivalent $\{211\}$ faces. Because the faceting phenomenon is a thermodynamic process, the nanopyramidal structure due to 
(a)

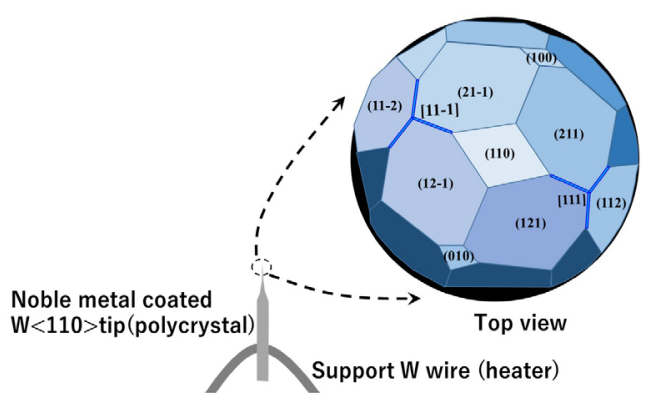

(b)

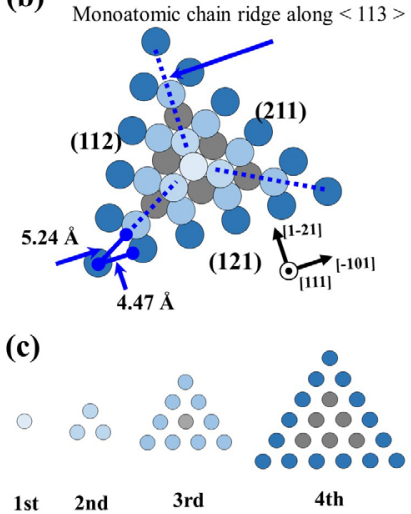

Figure 1: Schematic illustration of noble metal (NM)-coated W nanopyramid formed on W tip in the $\langle 110\rangle$ direction: (a) top view and (b) the hard-sphere model of three-sided pyramid, which is sided with equivalent $\{211\}$ planes surrounded with the $\langle 111\rangle$ poles. (c) Schematic of the stacked structure of the NM-coated W nanopyramid. The blue and gray circles represent the NM and W atoms, respectively.

faceting on W surface with thin adsorbate films is thermodynamically stable, and field emission microscopic studies suggesting the formation of such nanostructures have been reported by Sendecki and Barwinski in 1998 [24]. Inspired by the thermodynamical nature of the adsorbate-induced faceting process, Fu et al. [25] fabricated a Pd-coated W nanopyramid by depositing a Pd monolayer film on a carefully cleaned W tip and successfully revealed that the nanopyramid has a single atom termination and well-defined atomic stacking for several atomic layers via field ion microscopy (FIM). The hard-sphere model of the ideal nanopyramid is shown in Figure 1(b). The nanopyramid structure is characterized by three-sided $\{211\}$ facets separated by monoatomic chain ridges along the $\langle 113\rangle$ direction. The nanopyramid is comprised of the $\mathrm{W}$ atoms with a thin adsorbate metal film, rather than being pure adsorbate clusters, and are chemically inert. Tao et al. [21, 22] observed by high-resolution photoelectron spectroscopy that the $\mathrm{W} 4 \mathrm{f}_{7 / 2}$ core level (very sensitive to the chemical environment surrounding the $\mathrm{W}$ atoms) was shifted to the lower binding energy side of the W bulk peak that accompanied the W (211) facet formation on a face-centered cubic metal adlayer-adsorbed W(111) surface. According to previous studies, the stacking model of the nanopyramid is shown in Figure 1(c); the top terminated with a single NM atom, the second layer comprises three NM atoms (blue circles), the third layer comprises 10 atoms with a centered $\mathrm{W}$ atom (gray circles) surrounded by $9 \mathrm{NM}$ atoms, and the fourth layer comprises 21 atoms with 6 centered $\mathrm{W}$ atoms surrounded by 15 NM atoms [21, 22, 25, 26]. The nanopyramid has remarkable characteristics that enable its usage as an electron source, which includes high brightness [27-29], demountable character [27, 29], self-repairing function [25, 27], and low current fluctuation $[28,29]$. Because of these characteristics, nanopyramids are expected to be a next-generation coherent electron source.

Methods for supplying NM atoms are crucial for nanopyramid fabrication. Two methods for fabricating nanopyramids have been developed: Fu et al. [25] fabricated a
Pd-coated W nanopyramid via vacuum deposition, and Kuo et al. [28] proposed a technique involving electroplating deposition. In the electroplating method, NM atoms were supplied to $\mathrm{W}$ tips only by electroplating under an air atmosphere and masking with conventional nail polish to limit the amount of deposited NM films (hereinafter, "electroplating method"). Therefore, the electroplating method is simpler than the vacuum deposition method. An illustration of the electroplating method based on Kuo et al. [30] is shown in Figure 2. Ishikawa et al. [29] compared the vacuum deposition method with the nail-polish electroplating method and found that the field-emitted current stability of nanopyramids fabricated using the vacuum deposition

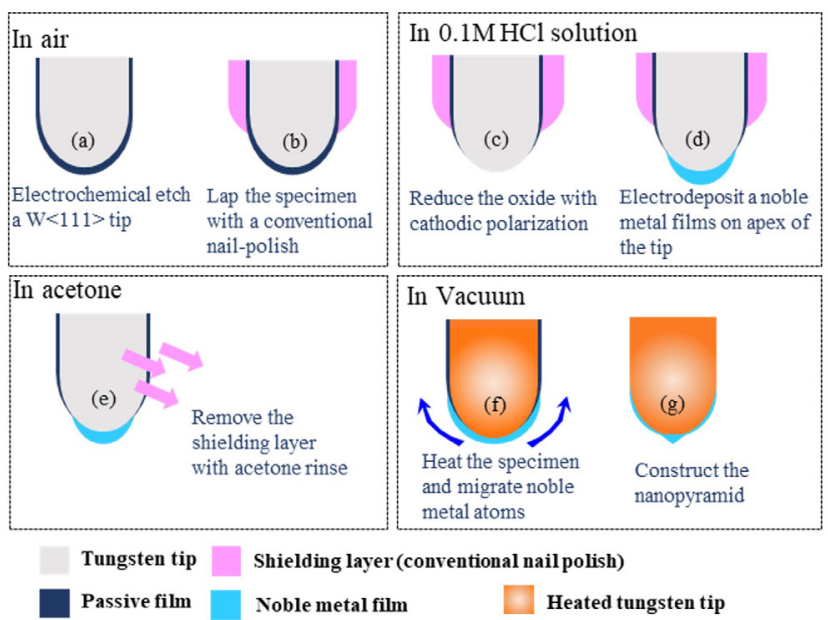

Figure 2: Schematic of the fabrication of noble metal (NM) source in the $\mathrm{W}$ tip end using direct electroplating. (a) The $\mathrm{W}$ tip sharpened via electropolishing. (b) Top of the $\mathrm{W}$ tip lapped with nail polish to limit the amount of deposited NM film. (c) Reduction of passive film by negative polarization in $0.1 \mathrm{M} \mathrm{HCl}$ solution. (d) Dropping an aqueous NM chloride solution to electrodeposit the NM film on the apex of the $\mathrm{W}$ tip. (e) Rinsing of the $\mathrm{W}$ tip with acetone to remove the nail polish. (f) Heating of the $\mathrm{W}$ tip in the UHV. The NM atoms diffuse and coat the surface of the W tip. (g) Nanopyramids fabricated at the apex of the $\mathrm{W}$ tip. 


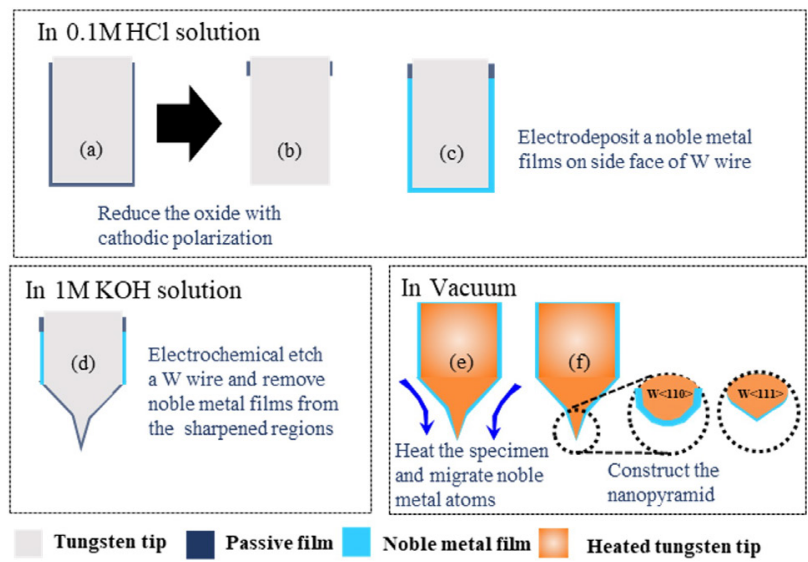

Figure 3: Schematic of the fabrication of noble metal (NM) source on the backward side of tungsten (W) tip using the surface diffusion method. (a) Cutting of the $\mathrm{W}$ wire into 5-mm lengths. (b) Reduction of the passive film by negative polarization in $0.1 \mathrm{M} \mathrm{HCl}$ solution. (c) Dropping an aqueous NM chloride solution to electrodeposit the NM film on the side surface of the entire W tip. (d) Sharpening the $\mathrm{W}$ tip by electropolishing. (e) Introduction of the $\mathrm{W}$ tip to the UHV chamber and heating it in the UHV environment. The NM atoms migrate from the backward side of the $\mathrm{W}$ tip and coat the apex of the $\mathrm{W}$ tip. (f) Nanopyramids fabricated at the apex of the $\mathrm{W}$ tip.

method was superior to that of nanopyramids fabricated by the electroplating method. By Auger electron spectroscopy, they found that residual impurities remained on the $\mathrm{W}\langle 111\rangle$ disc surface subjected to the same treatment used in the electroplating method and suggested that these impurities originated from the nail polish. To address residual impurities resulting from electroplating, Nakagawa et al. [31] recently proposed a novel method in which electroplated metal films were deposited on a back region far from the top of the ends, and NM atoms moved from the back to the front through a thermally activated surface diffusion process, similar to the method employed for fabricating conventional $\mathrm{ZrO} / \mathrm{W}$ Schottky emitters. As such, this process is called the "surface diffusion method." An illustration of the surface diffusion method is also shown in Figure 3. Interestingly, the field-emitted current stability of nanopyramids fabricated using the surface diffusion method was found to be comparable to that fabricated using the vacuum deposition method. Therefore, the surface diffusion method could be an ideal process for fabricating clean nanopyramids with improved current stability. In the surface diffusion method, the surface migration of the NM atoms on a W surface is necessary, which is because each NM has a different diffusion coefficient on the $\mathrm{W}$ surface, making the optimum $\mathrm{NM}-\mathrm{W}$ combination still unclear. Other conceivable criteria for determining the optimum NM-W combination include the binding energy of the top-most atom of the nanopyramid [26], NM-W binary state phase diagram [32], and anisotropy of the surface free energy of the NM-W system [23].

Therefore, in this study, we fabricate nanopyramids by electroplating $\mathrm{Pt}$ and $\mathrm{Au}$ films (as an NM other than $\mathrm{Pd}$ ) onto the rear region far from the end of a $\mathrm{W}$ tip by following a previous study [26]. Notably, the potential on the sample surface that controlled the electroplating reaction was unclear since the electroplating process was performed in a two-electrode electrochemical cell comprising only a working electrode (sample) and a counter electrode (carbon rod) in Ref. 26. This issue is addressed via a three-electrode electrochemical cell using a potentiostat with a reference electrode. Accordingly, we investigated the structural evolution via FIM and compared structural changes among Pt- and $\mathrm{Au}$-coated $\mathrm{W}$ tips. In addition, we investigated the regeneration process of destroyed nanopyramids and encountered regeneration failures during repeated regeneration. Further, to address the problems associated with the yield ratio for successful growth and the number of repetitions of nanopyramid regeneration, we examined the influence of heat on the NM-coated W tip in an electric field. The spatial distribution of field-emitted electrons has also been investigated by field-emission microscopy (FEM).

\section{EXPERIMENTAL}

All experiments were conducted in an ultra-high vacuum (UHV) chamber equipped with an extractor electrode and a chevron-type microchannel plate for FIM and FEM. The chamber's base pressure was set to be about $2 \times 10^{-8} \mathrm{~Pa}$. The extractor electrode was grounded and the specimen was positively biased during the FIM observations. Similarly, during the FEM observations, extractor electrode was posi-

(a) 1.Pre-etching for cleaning

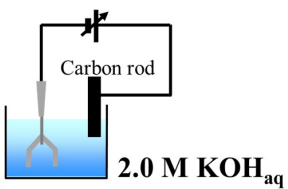

(b) 2.Electroplating for NM film deposition

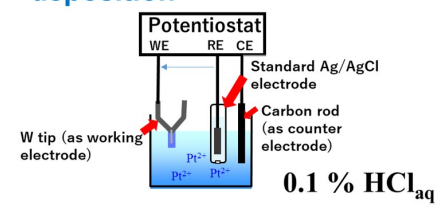

(c) 3.Etching for sharpening

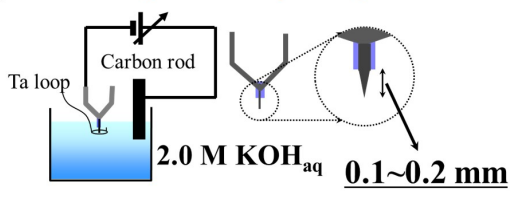

Figure 4: Schematic of manufacturing setup of the W tip with a NM reservoir. (a) Pre-etching process. The entire specimen is immersed in the electrolyte, and the thin skin is electrochemically removed. (b) Electroplating equipment. (c) Common chemicaletching process. 
tively biased, and the specimen was connected to the ground via an electrometer.

Figure 4 shows the fabrication process of the $\mathrm{W}$ tip with an NM reservoir. Polycrystalline $\mathrm{W}$ wires with a diameter of $0.25 \mathrm{~mm}$ were used to fabricate specimens. As a preliminary step, the specimens were chemically treated [Figure 4(a)]. The raw $\mathrm{W}$ wires were covered with native oxide and presumably contaminated by carbons or $\mathrm{Cu}$ particles due to the spot-weld process. To remove these contaminants, the specimens were completely immersed in $2 \mathrm{M} \mathrm{KOH}$ solution and kept at a positive voltage for few minutes. As a result, the diameter of the $\mathrm{W}$ wires was reduced to less than $0.2 \mathrm{~mm}$ and thereby, the new surfaces were obtained. Figure 5 shows energy-dispersive X-ray spectroscopy (EDX) spectra of the welded part of the specimen with (blue line) and without (violet line) electrochemical treatment. The electron beam was scanned in the range of $350 \mu \mathrm{m} \times 350 \mu \mathrm{m}$ and was accelerated at $20 \mathrm{keV}$. Note that the major peak of the Al-K line in Figure 5 essentially originated from the jig that holds the specimen. In the specimen case without the electrochemical treatment, a W-M line, a W-L line, and a weak $\mathrm{Cu}-\mathrm{K}$ line appear in the EDX spectra (violet line in Figure 5). We recognized large $\mathrm{Cu}$ particles with a size of around 150 $\mu \mathrm{m}$ on the secondary electron image of the same scanning area. The amount of $\mathrm{Cu}$ contamination is serious compared to the amount of the NM reservoir deposited by the electroplating process. Due to the electrochemical treatment, the $\mathrm{Cu}-\mathrm{K}$ line is disappeared, suggesting that the new surface of the specimen has been generated.

Subsequently, we deposited the $\mathrm{Pt}$ or $\mathrm{Au}$ films as reservoirs on the obtained new surface of the $\mathrm{W}$ tips by electroplating [Figure 4(b)]. Using a potentiostat, the electroplating process was performed in a three-electrode electrochemical cell. The electrochemical cell was composed of the $\mathrm{W}$ tip as the working electrode, a carbon rod as the counter electrode and a silver chloride electrode $(\mathrm{Ag} / \mathrm{AgCl})$ as a reference electrode. First, the $\mathrm{W}$ tip with about $5 \mathrm{~mm}$ long was immersed in $50 \mathrm{~mL}$ of $0.1 \% \mathrm{HCl}$ solution and held at $-0.6 \mathrm{~V}$ (vs. $\mathrm{Ag} / \mathrm{AgCl}$ ) for about $3 \mathrm{~min}$ to reduce the passive films.

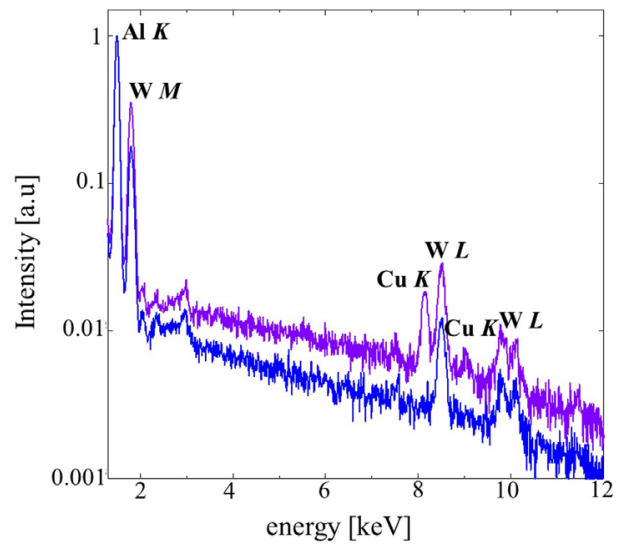

Figure 5: EDX spectra of the welded part of the specimen with (blue curve) and without (violet curve) pre-etching. The intensity of the Al-K line normalizes these spectra.
Subsequently, a small amount of $\sim 20 \mu \mathrm{L}$ of $1 \mathrm{mM} \mathrm{PtCl}_{2}$ solution was dropped for around $3 \mathrm{~min}$ into the electrochemical cell under the same cathodic polarization conditions. The deposition of the NM films on the W tip surfaces was confirmed by monitoring the Faradaic current. Figure 6 shows a typical Faradaic current evolution during electroplating with (blue curve) and without (red curve) the NM solution. The background current was found to be in the range of several to a dozen microamperes (i.e., from -3.9 to $-13 \mu \mathrm{A}$ ) during reducing the passive films ( 0 to $180 \mathrm{~s}$ ). Then, a drop of $\mathrm{PtCl}_{2}$ solution was added to the electrochemical cell, and consequently, the current was drastically increased to $-84 \mu \mathrm{A}$ and finally reached $-134 \mu \mathrm{A}$.

In contrast, the rapid increase in faradaic current from the background current was not observed when NM solution was not added to the cell. The number of Pt atoms deposited was estimated from Faradaic current measurement, which was found to be around $7.1 \times 10^{-8} \mathrm{~mol}$. It is surmised that the Pt films were formed uniformly over the entire surface of the $\mathrm{W}$ wire, and the thickness of the Pt films was calculated to be around $267 \mathrm{~nm}$.

The Pt-coated $\mathrm{W}$ wires were subsequently subjected to the $\mathrm{KOH}$ etching process [Figure 4(c)]. As a result, the Pt film was coated onto the $\mathrm{W}$ wire surfaces far from the tip end, whereas it was removed from nearby tip-end regions. To fabricate an $\mathrm{Au} / \mathrm{W}$ tip, instead of the $\mathrm{PtCl}_{2}$ solution, $1 \mathrm{mM}$ $\mathrm{AuCl}_{3}$ solution was used to prepare the $\mathrm{Au}$ films on the sides of the $\mathrm{W}$ tip.

The specimens thus obtained were introduced into the UHV chamber and annealed at $1000 \mathrm{~K}$, which is an appropriate temperature for the growth of the NM-coated nanopyramid $[25,26]$. In the UHV chamber, the $\mathrm{W}$ tips were cooled down to $100 \mathrm{~K}$, and FIM was used to observe the atomic structure of the tip-end. The $\mathrm{He}$ or $\mathrm{Ne}$ gas was introduced as an imaging gas at a pressure of approximately $2 \times$ $10^{-3} \mathrm{~Pa}$ via a variable leak valve during the FIM operation.

Before the heat treatment in the UHV, several atomic lay-

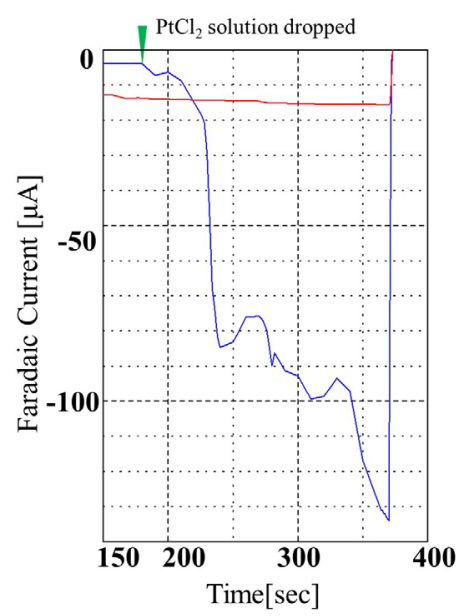

Figure 6: Typical Faradaic current evolution during electroplating with NM solution drop (blue curve) and without electroplating (red curve). 
ers at the apex of the specimen were removed by electric field evaporation. In the field evaporation method, the voltage $\left(V_{\text {tip }}\right)$ applied to the specimen during the FIM observation was increased to about two times the best image voltage (corresponding to the best image electric field of $\mathrm{He}$ or $\mathrm{Ne}$ ). Surface atoms were removed in the form of positive ions when the electric field strength on the tip exceeded the threshold value (which is called the critical evaporation field) as the voltage rises. Since the depth $(0.1 \mathrm{~nm}$ or less) of electric-field penetration into the bulk of metals was less than the spacing of the lattice plane [33], the field evaporation of metals started from the edges of a surface layer. As a result of the electric-field evaporation, the surface of the apex of the specimen became a smooth hemisphere shape. This technique is also applied to deliberately destroy the nanopyramid as described in Sec. III.B. The size of the substrate tip (a radius of curvature) is an essential factor for facet formation at the apex of the metal tip. It is presented that the nanopyramid can be formed at the end of the $\mathrm{W}$ tip with a curvature radius of at least $13-20 \mathrm{~nm}$ in our previous brief report [34]. In the FIM operation, the curvature radii of the specimens were estimated as follows using the "ring counting method" [33] to count the number of atomic steps from the (110) plane and the $\{211\}$ pole. In the ring counting method, the curvature radius of the specimen $(r)$ and the mean number of the atomic steps from the (110) plane to the $\{211\}$ pole $(n)$ are related as

$$
r=\frac{n s}{1-\cos \theta}=1.667 n(\mathrm{~nm}),
$$

where $s$ is the lattice spacing of the (110) planes, and $\theta$ is the angular separation between the (110) plane and the $\{211\}$ pole. The values of $s$ and $\theta$ were estimated to be $0.223 \mathrm{~nm}$ and $30^{\circ}$, respectively [33].

Finally, the tips were resistively heated through a $\mathrm{W}$ loop for mounting the tip, and the tip temperatures were measured using an optical pyrometer from the outside of the
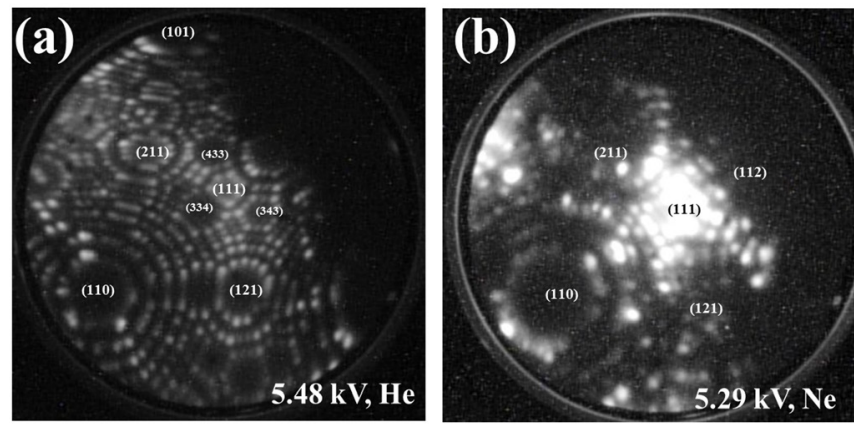

Figure 7: Typical field ion micrographs of the clean surface of a polycrystalline $\mathrm{W}$ tip. (a) Clean surface of polycrystalline $\mathrm{W}$ tip prepared by the field evaporation method (not heated). (b) A typical structural evolution result of a continuous heating treatment at $1000 \mathrm{~K}$.
UHV chamber. Figure 7 shows a typical FIM image at the best image electric field on the clean surface of a polycrystalline W tip (without the NM reservoir) and a typical structural change results of annealing at $1000 \mathrm{~K}$. Figure 7(a) shows that the typical arrangement of crystallographic faces with body-centered-cubic structure comprises low-index planes, such as $\{110\},\{111\},\{100\}$, and $\{211\}$. In Figure 7 (b), three $\{211\}$ faces are outspread, and a triangular base is formed on the $\{111\}$ faces. The $\{211\}$ plane expansion phenomenon is because the $\mathrm{W}$ atoms migrate via surface diffusion and expand the crystal plane with a low surface energy to reduce the free energy of the entire system [35, 36]. Because the free energy of the system is the product of the surface area and the surface energy, facet formation with an increase in the area is limited for pristine metal surfaces with a small surface energy anisotropy. In fact, as shown in Figure 7(b), three ridges separating neighboring $\{211\}$ faces are truncated due to insufficient expansion of the $\{211\}$ faces. In addition, the dark field-ion (FI)-beam spots scattered over the entire screen indicates that the anisotropy of the electric field at the $\{111\}$ pole is small, i.e., the $\{111\}$ faces do not protrude much toward the vacuum. Contrary to the thermal evolution of the $\{211\}$ faces of the clean W tip, the $\{211\}$ faces of the NM-coated W tip undergo extreme expansion. Because of the extreme faceting of the neighboring $\{211\}$ planes, the ridges that separate the $\{211\}$ planes become a straight array of atoms (monoatomic chain). For instance, the typical FI-micrograph of a single-atom termination, the second layer terminated with three atoms (trimer), and three ridges separating two adjacent $\{211\}$-side faces are shown in Figure 8. By increasing the tip voltage, the single-atom termination was removed by electric field evaporation, and the second layer terminated with three atoms was revealed [Figure 8 (b)]. By further careful increase in the tip voltage, the linear atomic chains between the neighboring $\{211\}$ facets formed concentric triangles [Figure 8 (c)]. In this study, the formation of nanopyramids was judged using the observation of monatomic chain ridges as an indicator. (a)

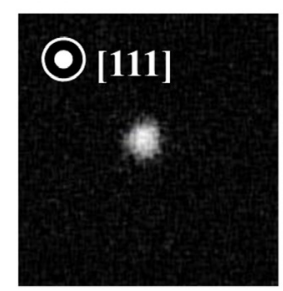

Single

(1st) (b)

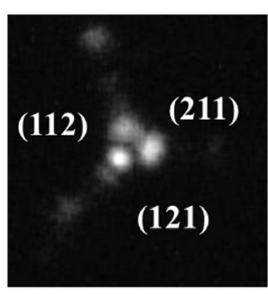

Trimer

(2nd) (c)

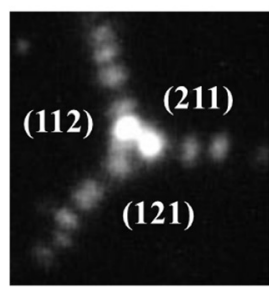

Mono atomic chain
Figure 8: Typical field ion micrographs of (a) single atom termination, (b) a second layer terminated with three atoms (trimer), and (c) three ridges separating two adjacent $\{211\}$-side faces. 


\section{RESULTS AND DISCUSSION}

\section{A. Atomic structure of Pt- or Au-coated nano- pyramids grown by surface diffusion method}

In this section, the structural evolution of a Pt- or Au-coated $\mathrm{W}$ tip caused under the heating treatment at 1000 $\mathrm{K}$ is demonstrated. First, to examine the effect of the NM adsorbate on the structural evolution of the $\mathrm{W}$ tip, the result corresponding to "without electroplating" in Figure 6 was observed, which indicated the structural evolution of the W tip without the NM source. Figure 9 shows the FI-micrographs indicating the structural evolution of the $\mathrm{W}$ tip without electroplating. Figure 9(a) depicts a He-FI-micrograph of a clean surface of the $\mathrm{W}$ tip obtained via a field evaporation technique. The curvature radii of the specimens were estimated to be around $11 \mathrm{~nm}$. Figure 9(b) depicts a Ne-FI-micrograph obtained after annealing for $270 \mathrm{~min}$. As a result of annealing for $270 \mathrm{~min}$, the $\{211\}$ surface surrounding the (111) pole was extended, but the ridge of the monatomic chain was unobserved, which meant that the $\mathrm{W}$ tip was not covered by an NM film (Figure 8).

In the case of the without-electroplated W tip, since no adsorbent is present on the tungsten surface, the $\{211\}$ facets are poorly developed, and nanopyramids were not formed on the W tip. Figure 10 presents the FI-micrographs indicating the structural evolution of Pt-coated W tip. Figure 10(a) was projected using $\mathrm{He}$ ions, and the other micrographs were projected using $\mathrm{Ne}$ ions. Notably, it was observed that $\mathrm{Pt}$ films did not cover the region around the apex of the specimen. From Figure 10(a), the number of the atomic steps from the (110) face to the (121), (12-1), (21-1), and (211) faces are estimated to be 8, 7, 9, and 7, respectively, and the curvature radius of the specimen was estimated to be around $13 \mathrm{~nm}$. Panels (b) and (d) of Figure 10 are the sample images obtained by subsequent heating for 10 and $90 \mathrm{~min}$, respectively. Notably, the comparison of micrographs [Figure 10(a,b)] reveals that the initial heat treatment resulted in the faceting of the $\{211\}$ faces, causing three ridges along the boundaries. Since it is necessary to increase the temperature higher than $1500 \mathrm{~K}$ to extend the $\{211\}$ fac-
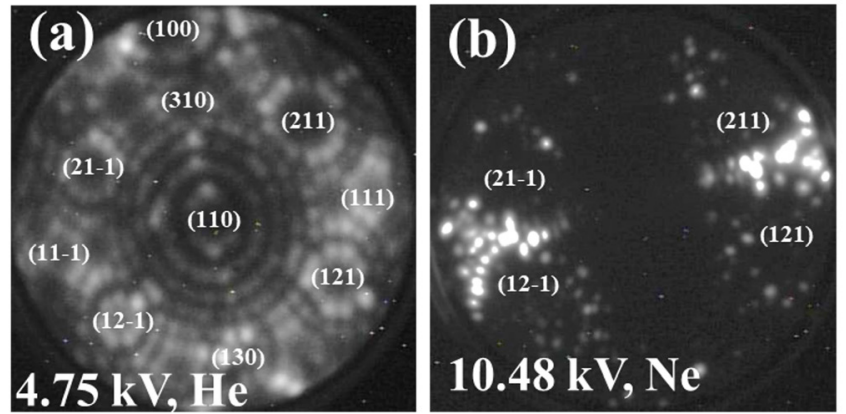

Figure 9: FI-micrographs of structural evolution of the W tip without the NM reservoir. (a) Subjected to a field evaporation treatment and not to annealing. (b) After the tip was annealed at $1000 \mathrm{~K}$ for $270 \mathrm{~min}$. et in the case of clean tungsten surface [36], this structural change at $1000 \mathrm{~K}$ indicated that there were numerous $\mathrm{Pt}$ atoms migrated from the Pt reservoir to get around the apex of the $\mathrm{W}$ tip. The additional annealing for $270 \mathrm{~min}$ was only one bright spot that corresponds to a single atom recognized in the FI-micrograph [Figure 10(d)]. When this atom was field-evaporated, the underneath layer that consisted of three atoms was emerged [Figure 10(e)]. Additionally, when the three-atom layer was field-evaporated, three monoatomic chain ridges directed along the equivalent $\langle 311\rangle$ direction was recognized [Figure 10(f)]. The pyramidal structures shown in Figure $10(\mathrm{~d}-\mathrm{f})$ are similar to the nanopyramid structure reported in previous studies [25, 27]. Therefore, it can be confirmed that the Pt-coated W tip with a single atom termination can be prepared using the surface diffusion method.

Figure 11(a, b) represents the FI-micrographs showing the structural evolution of the Au-coated W tip. Figure 11(a) was obtained in the He atmosphere, and the remaining images were obtained in the $\mathrm{Ne}$ atmosphere. Figure 11(a) shows the clean surface of the $\mathrm{W}$ tip. The number of atomic steps from the (110) face to the (121), (12-1), (21-1), and (211) faces are estimated to be 10, 7, 9, and 7, respectively. Accordingly, the curvature radius was calculated to be 13.7 $\mathrm{nm}$. Subsequently, the structure of the Au-coated W tip was subjected to changes through annealing treatments at 1000 $\mathrm{K}$ for 30,90 , and $180 \mathrm{~min}$ [Figure 11(b-d)]. Eventually, in the case of the Au-coated W tip, the nanopyramidal structure with three monoatomic chain ridges was formed, but the apex of the nanopyramid was not terminated with a single atom top. Such unusual behavior of the Au-coated nanopyramids was observed in previous studies as well in which the preparation methods other than the surface diffusion method were used [37]. The top-most $\mathrm{Au}$ atom was fieldevaporated during the FIM observation. Because the critical
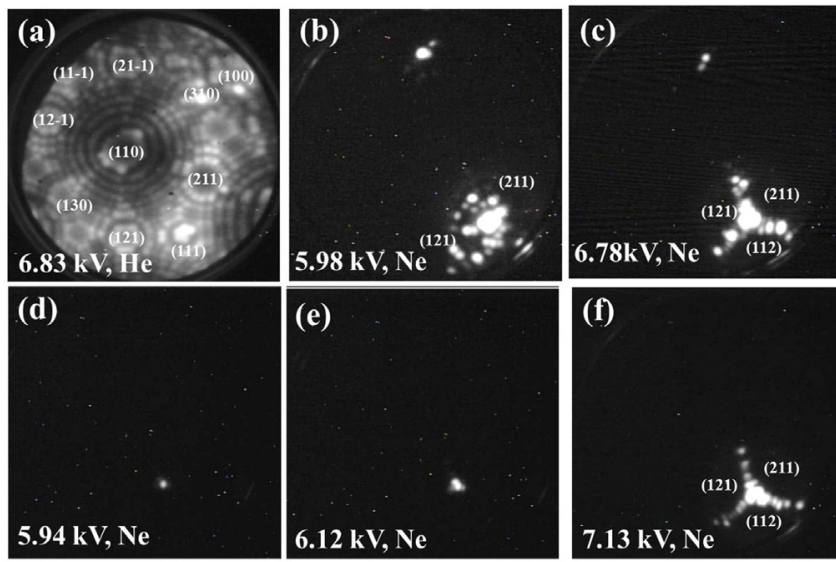

Figure 10: FI-micrographs of a clean Pt/W tip prepared via a surface diffusion method. The specimen (a) was subjected to a field evaporation treatment and not to annealing. After the tip was annealed at $1000 \mathrm{~K}$ for (b) $10 \mathrm{~min}$, (c) $90 \mathrm{~min}$, and (d)-(f) $270 \mathrm{~min}$. The single-atom top, trimer top, and three ridges were observed. 

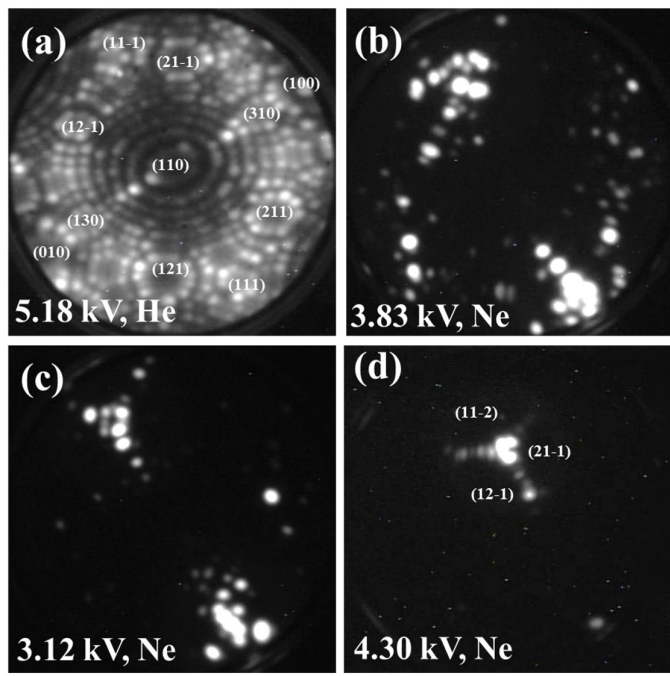

Figure 11: FI-micrographs showing the formation of the nanopyramid on the $\mathrm{Au} / \mathrm{W}$ tip. Images from (a) to (d) are arranged in order of administered treatments: (a) FIM of a clean $\mathrm{Au} / \mathrm{W}$ tip and (b) FI-micrograph observed after the sample in (a) was annealed at $1000 \mathrm{~K}$ for $30 \mathrm{~min}$. After the tip was annealed at $1000 \mathrm{~K}$ for (c) 90 min and (d) $180 \mathrm{~min}$. Three ridges were observed.

evaporation field of $\mathrm{Au}$ is $35 \mathrm{~V} \mathrm{~nm}^{-1}$, which is lower than the best image field of $\mathrm{Ne}$, which is $37.5 \mathrm{~V} \mathrm{~nm}^{-1}$ [33]. In fact in the Au-coated $\mathrm{W}$ tip, the atoms that construct the nanopyramid were gradually field-desorbed during the FIM operation. Due to the relatively low evaporation field, the robustness of the Au-coated nanopyramid is lower than that of the Pt- and Pd-coated nanopyramid as presumed in Ref. 37.

Table 1 marshaled the time for the nanopyramid formation of some Pt- and Au-coated W tips. Comparing the growth process of the Pt- and Au-coated W nanopyramids, the Au-coated $\mathrm{W}$ nanopyramid tends to take less time for its formation than when taken formation of the Pt-coated $\mathrm{W}$ nanopyramid. This nature can be explained by the difference in the surface diffusion of the NM atoms on tungsten. Assuming that surface diffusion is isotropic, then the diffusion time $t$ can be expressed as given in Eq. (1):

$$
t\left(E_{\mathrm{d}}, T\right)=\frac{1}{v} \propto \exp \frac{E_{\mathrm{d}}}{k_{\mathrm{B}} T},
$$

where $v$ is the frequency of hopping motion of adatoms, $E_{\mathrm{d}}$ is the activation energy for surface diffusion, $k_{\mathrm{B}}$ is the Boltzmann constant, and $t$ and $T$ are the time and temperature, respectively. According to literature, the values of $E_{\mathrm{d}}$ for Pt- and Au-atoms on the W(110) planes are $0.67 \mathrm{eV}$ [38] and $0.48 \mathrm{eV}$ [39], respectively. However, the ratio of the actual growth times for the Pt- and Au-coated nanopyramids is smaller than that of the one which is predicted from Eq. (1); $t_{\mathrm{Pt} / \mathrm{W}} / t_{\mathrm{Au} / \mathrm{W}}=\exp [(0.67-0.48) \mathrm{eV}] /(1000 \mathrm{~K} \times 8.617$ $\left.\times 10^{-5} \mathrm{eV} \mathrm{K}^{-1}\right)=9.1$, while $t_{\mathrm{Pt} / \mathrm{W}} / t_{\mathrm{Au} / \mathrm{W}}=t_{\text {tip A }} / t_{\text {tip B }}=$ 6.0 in our experimental results (Table 1). The reason for this variance is currently unknown, but it is convenient for this method to supply the NMs via the surface diffusion process. Elucidation of the factors that dominate the time required for
Table 1: The time required for the Pt and Au nanopyramid formation and the initial curvature radii.

\begin{tabular}{llcc}
\hline & NM/W & Time $(\min )$ & Curvature radius $(\mathrm{nm})$ \\
\hline Tip A & Pt/W & 540 & 13.3 \\
Tip B & Pt/W & 270 & 13.5 \\
Tip C & Pt/W & 420 & 12.9 \\
Tip D & $\mathrm{Au} / \mathrm{W}$ & 90 & 17.0 \\
Tip E & $\mathrm{Au} / \mathrm{W}$ & 180 & 12.5 \\
Tip F & $\mathrm{Au} / \mathrm{W}$ & 90 & 13.3 \\
\hline
\end{tabular}

nanopyramid growth is essential for controlling the nanopyramid formation and is a topic for future research.

\section{B. Validation of self-repairing function of the nanopyramid grown via a surface diffusion process}

The self-repairing function is a unique characteristic of the NM-coated nanopyramids that other nanotips unequipped with one do not possess, and it is crucial for its application in charged particle optical instruments. In this section, Pt- and Au-coated nanopyramids fabricated using the surface diffusion method were examined to assess whether they were equipped with the self-repairing function as nanopyramids fabricated by other methods. To do this, the fabricated nanopyramids were deliberately destroyed using two methods; namely, field evaporation and atmospheric air exposure methods. The field evaporation caused mild destruction, but it did not introduce any defects in the material
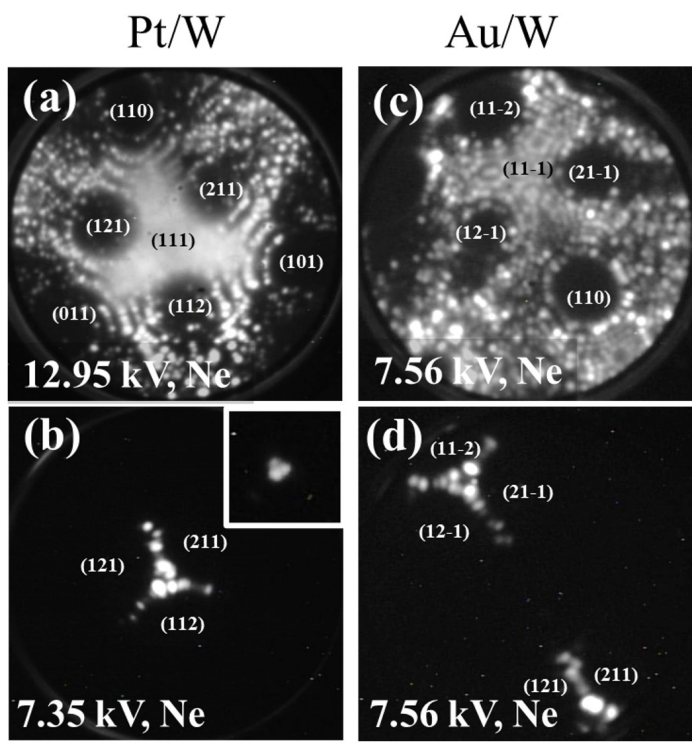

Figure 12: (a, c) FI-micrographs of Pt- and Au-coated W tips in which several atomic layers, including nanopyramids, were removed by electric field evaporation. (b) FI-micrograph observed after the sample in (a) was annealed at $1000 \mathrm{~K}$ for $30 \mathrm{~min}$. (d) FI-micrograph observed after the sample in (b) was annealed at $1000 \mathrm{~K}$ for $30 \mathrm{~min}$. 

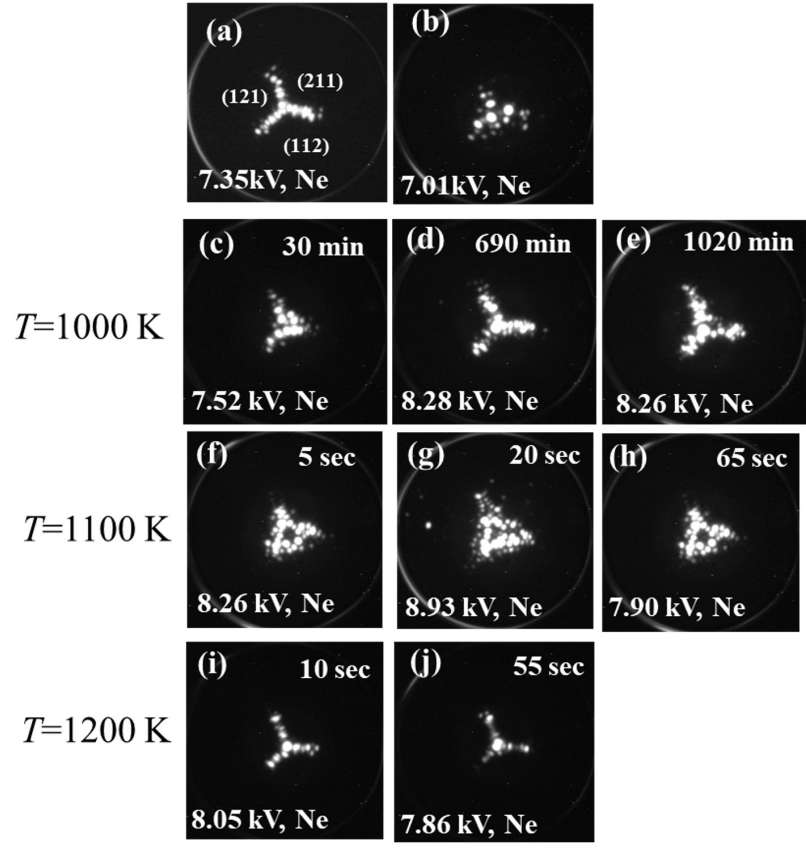

Figure 13: FI-micrographs of Pt-coated nanopyramid repair function test results after air exposure. (a) Initial fabricated, (b) intentionally destroyed using air exposure. The series of (c) to (e) represents results of the annealing treatment at $1000 \mathrm{~K}$. The series of (f) to (h) represents results of the annealing treatment at $1100 \mathrm{~K}$. (i) and (j) represents results of the annealing treatment at $1200 \mathrm{~K}$. Annealing time and the imaging voltage are noticed in each panel.

due to thermal disturbance, stress, and contamination with impurities [33]. Meanwhile, air exposure caused severe destruction accompanied by collision and adsorption of gas molecules, such as oxygen and water. Figure 12(a-c) shows Ne-FI-micrographs of the Pt- and Au-coated W tips in which several atomic layers, including nanopyramids, were removed by electric field evaporation. The homogeneous distribution of the FI-beam spots appeared over the entire fluorescence screen. Several layers at the apex of the tip were removed by the field evaporation process and changing its shape from polyhedral to hemispherical geometry. Figure 12(b, d) shows the results of heat treatments of the Pt- and Au-coated $\mathrm{W}$ tips in the UHV at $1000 \mathrm{~K}$. The FI pattern changed to a linear arrangement, indicating a monatomic chain, which confirmed the formation of nanopyramids.

Moreover, the FI-micrographs corresponding to the regeneration process of nanopyramids indicated their collapse by air exposure (Figures 13 and 14). The air exposure was done by taking the specimen outside the UHV chamber. Subsequently, the specimen was reintroduced to the UHV chamber and heated in the UHV environment. Figure 13(a, b) shows the FI-micrographs of the repair function test results corresponding to the Pt-coated nanopyramid that was initially fabricated and intentionally destroyed using air exposure. Panels $(\mathrm{c}-\mathrm{e}),(\mathrm{f}-\mathrm{h})$, and $(\mathrm{i}, \mathrm{j})$ of Figure 13 show the results of annealing treatments at 1000, 1100, and $1200 \mathrm{~K}$, respectively. Figure $13(\mathrm{f}-\mathrm{h})$ shows results of the annealing
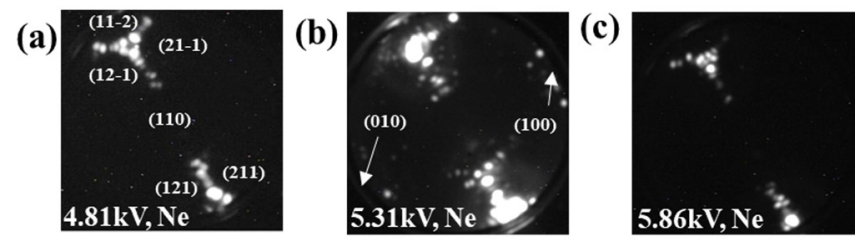

Figure 14: FI-micrographs of Au-coated nanopyramid repair function test results after air exposure: (a) an initially fabricated nanopyramid, (b) an intentionally destroyed nanopyramid using air exposure, and (c) a regenerated nanopyramid.

treatment at $1100 \mathrm{~K}$ for 5, 20, and $65 \mathrm{~s}$, and Figure $13(\mathrm{~g}, \mathrm{~h})$ shows the results of additional annealing treatment with the temperature increased to $1200 \mathrm{~K}$. Notably, the Pt-coated nanopyramid collapsed by air exposure was not regenerated at 1000 and $1100 \mathrm{~K}$. Contrastingly, the Pt-coated nanopyramid annealed at $1200 \mathrm{~K}$ successfully generated the monoatomic chain ridges [Figure 13(f)]. Meanwhile, in the Au-coated $\mathrm{W}$ tip, the nanopyramid was regenerated only by the annealing treatment at $1000 \mathrm{~K}$ after air exposure. Figure 14(a, b) represents the fabricated $\mathrm{Au}$ nanopyramids before and after air exposure. Figure 14(c) shows the result of the annealing treatment at $1000 \mathrm{~K}$ for $480 \mathrm{~min}$. Notably, in repeated regeneration experiments, the nanopyramids are always formed in the same site (i.e., around the $\langle 111\rangle$ pole) before they are destroyed. If the nanopyramids were clusters of Au or Pt atoms, they would form in random locations. We believe that the nanopyramids observed in this experiment are not clusters of adsorbed NM atoms, but they are the result of the surface reconstruction of the $\mathrm{W}$ substrate induced by adsorption of a thin NM film, similar to that reported by Madey et al. in the previous studies [18-22].

The structural evolution of the air-exposed Pt-coated W tip at 1000 and $1100 \mathrm{~K}$ is similar to "the pause in nanopyramid growth" phenomenon (i.e., the expansion of $\{211\}$ facets is stopped, and sharp ridges are not formed on the nanopyramid), which Nakagawa et al. [31] reported in the formation process of a Pd-coated nanopyramid at $1000 \mathrm{~K}$. Their study hypothesized that "the pause in nanopyramid growth" was due to the inhibition of the migration of $\mathrm{W}$ atoms caused by defects, grain boundaries, and extra-activation barriers from clusters when their kinetics were considered. The difference in structural changes between the $\mathrm{Au}$ - and Pt-coated W tips at $1000 \mathrm{~K}$ after air exposure suggested that the Pt/W system was more susceptible to defects than the $\mathrm{Au} / \mathrm{W}$ system, consistent with the kinetic considerations that the pause in nanopyramid growth of the Pt-coated $\mathrm{W}$ tip was resolved by increasing the heating temperature to $1200 \mathrm{~K}$. Although we encountered irregular cases, the uncertainty of structural change in the NM-coated nanopyramid, which underwent repeated cycles of collapse and self-repairing, did not resolve even at higher temperatures $(1200-1500 \mathrm{~K})$. This phenomenon essentially limits the lifetime of the nanopyramid and cannot be solved solely by kinetic techniques that facilitate atomic transfer at high temperatures. 


\section{Anchoring of NM-coated nanopyramid on W tip with ultimate sharpness under high- temperature and electrical polarizing}

In Sec. III.B, it is discussed that the monoatomic chain ridges are not formed even if the expansion of the nanopyramid with the stagnation of the $\{211\}$-side faces continues through annealing treatment. Accordingly, it is hypothesized that the stagnation of the $\{211\}$-side face expansion is not the only limit of mass transport but also the transfiguration of a thermal equilibrium crystal shape due to reduction of the surface-free-energy anisotropy (a driving force of $\{211\}$-side face faceting). There is a thermal electric field (TF) treatment to enhance the surface-free-energy anisotropy, where the metal tip is heated under a strong electric field. Sharpening of the W tip using the TF-treatment is traditionally called remolding or buildup, and there has been much research since the early times [40]. From a theoretical perspective, Fujita et al. have explained the sharpening of metal tips at high temperatures and under a strong electric field by the capacitor model [41]. In this capacitor model, the metal tip and the counter electrode are considered one closed system, and the shape of the metal tip is changed to minimize the Gibbs energy $G=F_{\mathrm{i}}-U_{\mathrm{E}}$ of the system, where $F_{\mathrm{i}}$ is the intrinsic Helmholtz free energy of the metal tip and $U_{\mathrm{E}}$ is the electric energy stored in the capacitor. Note that the value of $F_{\mathrm{i}}$ is anisotropic, while the value of $U_{\mathrm{E}}$ is isotropic. The shape of the metal tip in the absence of the field is determined by the surface free energy of the metal crystal, which
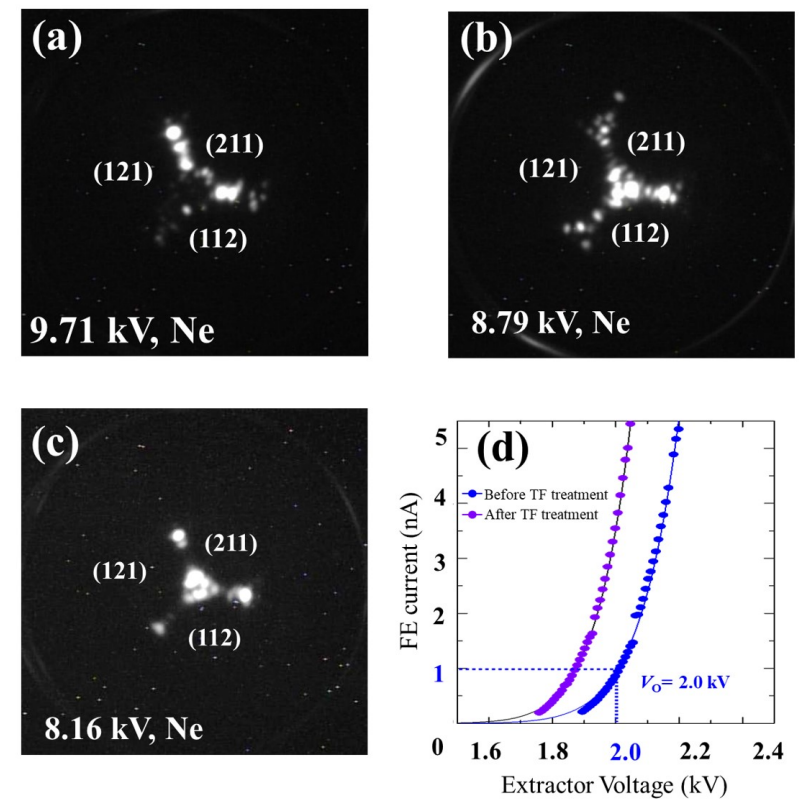

Figure 15: Changes in FI-micrograph and FE current versus extractor voltage curves due to heating at $1200 \mathrm{~K}$ simultaneously with the application of the electric field. (a) Pt-coated nanopyramid that stops an expansion of the $\{211\}$-facet sides. (b, c) FI-micrographs after the annealing treatment at $1200 \mathrm{~K}$ under $V_{\mathrm{R}} / V_{\mathrm{O}}=-1$ for $60 \mathrm{~s}$ (b) and $120 \mathrm{~s}(\mathrm{c})$. (d) $I-V$ curves of the nanopyramid that corresponds to (a) (blue curve) and (c) (violet curve). is derived from the intrinsic Helmholtz free energy $F_{\mathrm{i}}$. However, once the external field is applied to the metal tip, the metal tip begins to transform into the shape that can store higher electric energy $U_{\mathrm{E}}$. As a consequence, the shape of the metal tip apex changes from spherical to polyhedral. A uniform free energy reduction increasingly enhances the anisotropy of the surface free energy due to the electrostatic energy. As a result, the evolution of the facet formation is achieved on the apex of the metal tip. Recently, from the perspective of an experimental work conducted, Rokuta et al. reported the phase diagram of structural changes of the apex of a $\mathrm{W}\langle 111\rangle$ tip as a function for remolding temperature $T_{\mathrm{R}}$ versus normalized remolded voltage $V_{\mathrm{R}} / V_{\mathrm{O}}$, where $V_{\mathrm{O}}$ is defined as an extractor voltage required for the field-emission (FE) current of $1 \mathrm{nA}$ [42]. They clarified that the remolding treatment at $2100 \mathrm{~K}$ under $V_{\mathrm{R}} / V_{\mathrm{O}}=1$ induced $\{211\}$-faceting most significantly under all conditions they examined. Based on the electric field condition $\left(V_{\mathrm{R}} / V_{\mathrm{O}}=1\right)$, which was found in the works by Rokuta et al., we examined the effect of the TF-treatment for the nanopyramid with the stagnation of the $\{211\}$-side face expansion. Accordingly, an annealing temperature of $1200 \mathrm{~K}$ was chosen because $\mathrm{Pt}$ can be desorbed from the W tip at temperatures above 1900 $\mathrm{K}$ [43]. The structural changes of the Pt-coated nanopyramid due to the annealing treatment in the static electric field are presented in Figure 15. The Pt-coated nanopyramid has stopped expansion of the $\{211\}$-facet sides after annealing at $1000 \mathrm{~K}$ for $360 \mathrm{~min}$ as well as at $1200 \mathrm{~K}$, as shown in Figure 15(a). The blue and violet curves of the $I-V$ characteristic shown in Figure 15(d) corresponds to the nanopyramids in panels (a) and (b) in Figure 15, respectively. The onset voltage $V_{\mathrm{o}}$ required for the emission current of $1 \mathrm{nA}$ is $2 \mathrm{kV}$
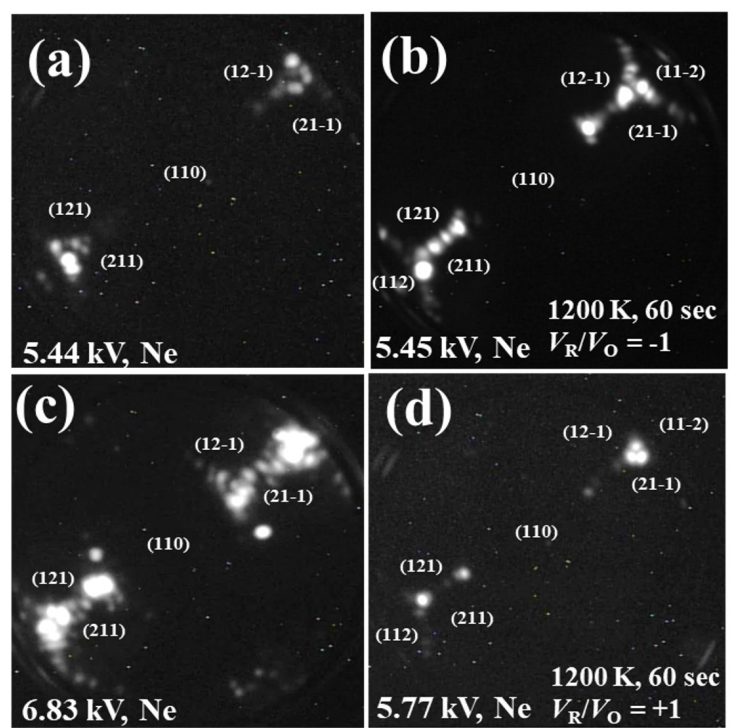

Figure 16: Dependence of the polarity of the electric field on the grown nanopyramid. (a, c) The nanopyramid are destroyed using air exposure. (b, d) The nanopyramid subjected to the annealing treatments at $1200 \mathrm{~K}$ under $(\mathrm{b})$ the positive bias $\left(V_{\mathrm{R}} / V_{\mathrm{O}}=-1\right)$ and (d) the negative bias $\left(V_{\mathrm{R}} / V_{\mathrm{O}}=+1\right)$. 
[Figure 15(d)]. Figure 15(b, c) shows the changes of the atomic structure of the sample annealed at $1200 \mathrm{~K}$ with the electric field of $V_{\mathrm{R}}=-2.0 \mathrm{kV}\left(V_{\mathrm{R}} / V_{\mathrm{O}}=-1\right)$ for 60 and $120 \mathrm{~s}$ done additionally on the specimen corresponding to that of Figure 15(a). After annealing for $60 \mathrm{~s}$, a bright-line separated between (121) and (112) appears in the FI-micrograph [Figure 15(b)]. This suggested the expansion of the (121) and (112) side faces. The additional TF-treatment for $60 \mathrm{~s}$ (total $120 \mathrm{~s}$ ) resulted in the regeneration of the nanopyramid with three equivalent monoatomic chain ridges and an apex terminated with fifteen atoms [Figure 15(c)]. Thus, it was predicted that the TF-treatment reduces the structural disorder on the surface of NM-coated W and makes it possible to grow as a nanopyramid structure easily. Figure 16 shows the polarity dependence of structural changes in the Au-coated nanopyramid with the stagnation of the $\{211\}$-side face expansion. The Au-coated nanopyramid with a truncated expansion of the $\{211\}$ facet after annealing at $1000 \mathrm{~K}$ for 240 min is shown in Figure 16(a). After the TF-treatment at 1200 $\mathrm{K}$ for $60 \mathrm{~s}$ in $V_{\mathrm{R}}=-1.0 \mathrm{kV}\left(V_{\mathrm{R}} / V_{\mathrm{O}}=-1\right)$, the nanopyramid formation was recognized as shown in the FI-micrograph in Figure 16(b). Subsequently, the nanopyramid was destroyed by air exposure, and the TF-treatment was done at $1200 \mathrm{~K}$ for $60 \mathrm{~s}$ in $V_{\mathrm{R}}=+1.3 \mathrm{kV}\left(V_{\mathrm{R}} / V_{\mathrm{O}}=+1\right)$ [Figure $16(\mathrm{c}, \mathrm{d})$ ]. Notably, regardless of the voltage polarity, the formation of the nanopyramid was achieved [Figure 16(d)]. Additionally, the Pt- and Pd-coated nanopyramid formation was independent of the voltage polarity during the TF-treatment in our experiment. We considered that the electric energy stored between the tip and the counter electrode is proportional to the square of the value of an electric field, and thereby the nanopyramid formation is independent of the voltage polarity in the TF-treatment. The rapid formation of the nanopyramid by the TF-treatment is shown in Figure 16, and the extrication of the stagnation of the $\{211\}$ side-face expansion happened due to the influence of the surface-free-energy anisotropy under the electric field. Recently, Oshima et al. have demonstrated the thermal stability of the Ir-coated W nanopyramid and reported that the Ir-coated nanopyramid formation was achieved only at negative biases above the observable one of the FE-micrographs ( $V_{\mathrm{R}}$ was positive) in a temperature range below $1300 \mathrm{~K}$ [44]. The difference in behavior between the Ir-coated nanopyramid and the Pt-, $\mathrm{Au}-$, and Pd-coated nanopyramids under electric fields is currently unknown. In any case, based on our experiment, we would like to emphasize that the TF-treatment is of great utility to the successful formation of the nanopyramid and prolonging the lifetime of the nanopyramid as a promising electron source in an enclosed environment such as a conventional electron gun.

\section{Comparison of FE patterns of Pt-, Au-, and Pd-coated nanopyramids}

Figure 17(a, c) shows FEM patterns of Pt- and Au-coated nanopyramids prepared using the surface diffusion method. During the FEM observation, the pressure raised to $7.0 \times$

\section{FEM FIM}
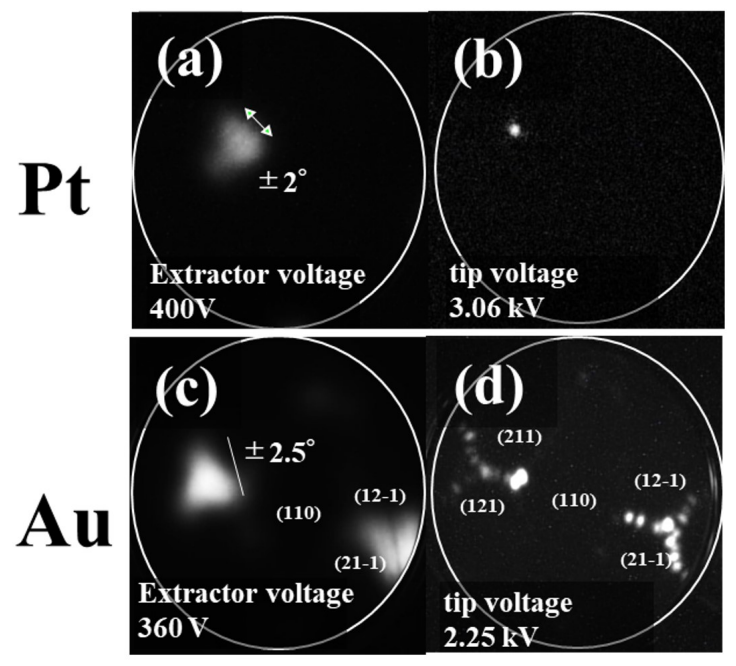

Figure 17: FEM patterns of (a) Pt-, (c) Au-coated nanopyramids. (b, d) FIM images corresponding to the FEM pattern are shown on the right. White circles represent the outer periphery of the fluorescent screen, whereas arrows and characters denote a measure of the opening angle of the field-emitted electron beam.

$10^{-8} \mathrm{~Pa}$. All the FEM patterns in Figure 13 exhibit triangular shapes, indicating the NM-coated nanopyramids, where it also shows that the structure at the pyramid top is not a single atom but rather one of the underneath layers with three-fold symmetry. This observation contradicts the corresponding FIM micrographs for Pt and Pd. Accordingly, it can be proposed that the single atoms observed in Figure 13(a, c) were not expected at the nanopyramid tip but rather accidentally appeared because of some adsorbates or migrating atoms. The opening angles of the field-emitted electrons were approximately $\pm 2^{\circ}$ and $\pm 2.5^{\circ}$ for Pt and $\mathrm{Au}$, respectively. The opening angles of the electron beams appeared to be wide for a single-atom top, similar to those observed for the trimer or decamer termination [28, 37, 45]. The FE patterns shown in Figure 17 suggest that the stable electron emission from the NM-coated nanopyramid requires an extra-high vacuum (XHV) condition $\left(<1 \times 10^{-9}\right.$ $\mathrm{Pa})$. To further characterize the FE properties, such as the probe current or the FE pattern's angular current density and the stability, future works should be conducted to investigate these properties in the electron-gun system by evacuating the XHV using a non-evaporable getter pump [46, 47]. Applying the XHV environment in future studies would give better insights into the concepts of electron optics performance of this NM-coated W nanopyramid.

\section{CONCLUSIONS}

This study can be concluded as follows.

1) W nanopyramids coated with monolayer films of Pt and $\mathrm{Au}$ were identical to those fabricated previously using other methods. The difficulty of fabrication was essen- 
tially similar for the Pt- or Au-coated nanopyramid.

2) Self-repairing of the Pt- and Au-coated nanopyramids destroyed by the field evaporation or air exposure was confirmed by additional annealing treatments. The repeated destruction and regeneration of the nanopyramid can cause the failure of the structure regeneration, i.e., the $\{211\}$-side faces do not expand even under the continued annealing treatment.

3) The NM-coated W tip that failed in regeneration was the one that simultaneously heated at $1200 \mathrm{~K}$ along with the application of the electric field. Notably, upon the applied electric field during the annealing treatment, the $\{211\}$ side face expansion was quickly achieved.

4) FE patterns were found to be essentially the same as those observed in the previous studies reported in literature with no recognizable distinctions. Further investigation is necessary to understand the FE characters toward its application for the practical electron source.

5) The fabrication method is a critical issue for the development of nanoscale FE sources for practical applications. Accordingly, we propose that the present method is less risk of contamination and facile.

\section{Acknowledgments}

The authors want to thank Professor Chuhei Oshima of Waseda University and Professor Hiroshi Shimoyama of Meijo University for their encouragement and advice. We acknowledge the help of Ms. Yamazaki, Mr. Sano, and Professor Hatsuhiko Usami of Meijo University, who provided the EDX experimental data of the samples. Hirotaka Asai is indebted to Dr. Shin Fujita of Shimadzu (Asia Pacific) for his suggestions on the TF treatment experiments during the Tenth International Conference on Charged Particle Optics. We are grateful to Mr. Asai, Mr. Kato, Mr. Suzuki, Mr. Nakane, Mr. Kawabe, Mr. Morita, Mr. Yamaguchi, Mr. Iida, Mr. Gotoh, Mr. Kawai, Mr. Matsubara, Mr. Ozeki, Mr. Mori, Ms. Sakoguchi, Mr. Yamazaki, Mr. Ogino, Mr. Mikawa, and Mr. Furusawa for their help in the FIM/FEM experiment. The authors would like to thank Enago (www.enago.jp) for the English language review.

\section{References}

[1] M. Kuwahara, S. Kusunoki, Y. Nambo, K. Saitoh, X. Jin, T. Ujihara, H. Asano, Y. Takeda, and N. Tanaka, Appl. Phys. Lett. 105 , 193101 (2014).

[2] K. Murakami, T. Igari, K. Mitsuishi, M. Nagao, M. Sasaki, and Y. Yamada, ACS Appl. Mater. Interfaces 12, 4061 (2020).

[3] S. Nagai, H. Ikemizu, K. Hata, and T. Ishikawa, J. Appl. Phys. 126, 135302 (2019).

[4] H. Zhang, J. Tang, J. Yuan, Y. Yamauchi, T. T. Suzuki, N. Shinya, K. Nakajima, and L.-C. Qin, Nat. Nanotechnol. 11, 273 (2016).

[5] K. Kasuya, T. Kusunoki, T. Hashizume, T. Ohshima, S. Katagiri, Y. Sakai, and N. Arai, Appl. Phys. Lett. 117, 213103 (2020).

[6] X. Shao, A. Srinivasan, W. K. Ang, and A. Khursheed, Nat. Commun. 9, 1288 (2018).

[7] S. Matsunaga and Y. Sohda, AIP Adv. 9, 065001 (2019).
[8] H. Shimoyama, A. Ohshita, and S. Maruse, Jpn. J. Appl. Phys. 11, 150 (1972).

[9] H. Shimoyama and S. Maruse, Ultramicroscopy 15, 239 (1984).

[10] H.-W. Fink, IBM J. Res. Dev. 30, 460 (1986).

[11] V. T. Binh and J. Marien, Surf. Sci. 202, L539 (1988).

[12] H. U. Müller, B. Völkel, M. Hofmann, C. Wöll, and M. Grun-

ze, Ultramicroscopy 50, 57 (1993).

[13] K. Nagaoka, H. Fujii, K. Matsuda, M. Komaki, Y. Murata, C. Oshima, and T. Sakurai, Appl. Surf. Sci. 182, 12 (2001).

[14] M. Rezeq, J. Pitters, and R. Wolkow, J. Chem. Phys. 124, 204716 (2006).

[15] F. Rahman, J. Onoda, K. Imaizumi, and S. Mizuno, Surf. Sci. 602, 2128 (2008).

[16] J. Onoda, S. Mizuno, and H. Ago, Surf. Sci. 604, 1094 (2010).

[17] J. Onoda and S. Mizuno, Appl. Surf. Sci. 257, 8427 (2011).

[18] T. E. Madey, J. Guan, C.-Z. Dong, and S. M. Shivaprasad, Surf. Sci. 287-288, 826 (1993).

[19] T. E. Madey, J. Guan, C.-H. Nien, C.-Z. Dong, H.-S. Tao, and R. A. Campbell, Surf. Rev. Lett. 3, 1315 (1996).

[20] T. E. Madey, C.-H. Nien, K. Pelhos, J. J. Kolodziej, I. M. Abdelrehim, and H.-S. Tao, Surf. Sci. 438, 191 (1999).

[21] H.-S. Tao, C.-H. Nien, T. E. Madey, J. E. Rowe, and G. K. Wertheim, Surf. Sci. 357-358, 55 (1996).

[22] H.-S. Tao, J. E. Rowe, and T. E. Madey, Surf. Sci. 407, L640 (1998).

[23] S. P. Chen, Surf. Sci. 274, L619 (1992).

[24] S. Sendecki and B. Barwinski, Appl. Surf. Sci. 134, 243 (1998).

[25] T.-Y. Fu, L.-C. Cheng, C.-H. Nien, and T. T. Tsong, Phys. Rev. B 64, 113401 (2001).

[26] H.-T. Jeng, H.-S. Kuo, I.-S. Hwang, and T. T. Tsong, Phys. Rev. B 81, 155424 (2010).

[27] E. Rokuta, T. Itagaki, T. Ishikawa, B.-L. Cho, H.-S. Kuo, T. T. Tsong, and C. Oshima, Appl. Surf. Sci. 252, 3686 (2006).

[28] H.-S. Kuo, I.-S. Hwang, T.-Y. Fu, J.-Y. Wu, C.-C. Chang, and T. T. Tsong, Nano Lett. 4, 2379 (2004).

[29] T. Ishikawa, K. Tagawa, T. Urata, C. Oshima, B. Cho, and E. Rokuta, e-J. Surf. Sci. Nanotechnol. 6, 11 (2008).

[30] H.-S. Kuo, I.-S. Hwang, T.-Y. Fu, Y.-C. Lin, C.-C. Chang, and T. T. Tsong, Jpn. J. Appl. Phys. 45, 8972 (2006).

[31] T. Nakagawa, E. Rokuta, H. Murata, H. Shimoyama, and C. Oshima, J. Vac. Sci. Technol. B 31, 02B105 (2013).

[32] T. B. Massalski, H. Okamoto, P. R. Subramanian, and L. Kacprzak (Eds.), Binary Alloy Phase Diagrams, 2nd ed. (ASM International, Materials Park, OH, 1990).

[33] E. W. Müller and T. T. Tsong, Field Ion Microscopy; Principles and Applications (Elsevier, New York, 1969).

[34] H. Asai, T. Tanaka, H. Murata, and E. Rokuta, e-J. Surf. Sci. Nanotechnol. 18, 185 (2020).

[35] M. Drechsler, in: Surface Mobilities on Solid Materials; Fundamental Concepts and Applications, edited by V. T. Bihn (Springer, Boston, 1983) p. 405.

[36] S. Nishigaki and S. Nakamura, Jpn. J. Appl. Phys. 15, 1647 (1976).

[37] K. Nomura, E. Rokuta, T. Itagaki, C. Oshima, H. Kuo, and T. T. Tsong, e-J. Surf. Sci. Nanotechnol. 6, 25 (2008).

[38] D. W. Bassett, J. Phys. C 9, 2491 (1976).

[39] J. P. Jones and N. T. Jones, Thin Solid Films 35, 83 (1976).

[40] P. C. Bettler and F. M. Charbonnier, Phys. Rev. 119, 85 (1960).

[41] S. Fujita and H. Shimoyama, Phys. Rev. B 75, 235431 (2007). 


\section{Regular Paper}

[42] E. Rokuta, T. Nakagawa, H. Murata, S. Fujita, H. Shimoyama, and C. Oshima, Jpn. J. Appl. Phys. 50, 115001 (2011).

[43] G. Antczak, T. E. Madey, M. Błaszczyszyn, and R. Błaszczyszyn, Vacuum 63, 43 (2001).

[44] C. Oshima, M. Tomitori, T. Shimoda, A. Yasaka, H. Asai, and E. Rokuta, e-J. Surf. Sci. Nanotechnol. 16, 294 (2018).

[45] E. Rokuta, H.-S. Kuo, T. Itagaki, K. Nomura, T. Ishikawa, B.-L. Cho, I.-S. Hwang, T. T. Tsong, and C. Oshima, Surf. Sci. 602, 2508 (2008).

[46] T. Ishikawa, T. Urata, B. Cho, E. Rokuta, C. Oshima, Y. Terui, H. Saito, A. Yonezawa, and T. T. Tsong, Appl. Phys. Lett. 90, 143120 (2007).

[47] K. Kasuya, S. Katagiri, T. Ohshima, and S. Kokubo, J. Vac. Sci. Technol. B 28, L55 (2010).

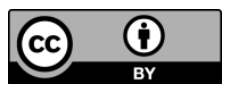

All articles published on e-J. Surf. Sci. Nanotechnol. are licensed under the Creative Commons Attribution 4.0 International (CC BY 4.0). You are free to copy and redistribute articles in any medium or format and also free to remix, transform, and build upon articles for any purpose (including a commercial use) as long as you give appropriate credit to the original source and provide a link to the Creative Commons (CC) license. If you modify the material, you must indicate changes in a proper way.

Published by The Japan Society of Vacuum and Surface Science 